\title{
Les Régulateurs d'induction ${ }^{(1)}$
}

Les régulateurs d'induction sont des régulateurs de tension; ils remplissent pour les courants alternatifs le rôle des survoltcurs-dévolteurs pour le courant continu, aussi les désigne-t-on parfois sous le nom de survolteurs à induction ou simplement de survolteurs. Ces appareils sont de construction très sensiblement identique à celle des moteurs asynchrones. Mais, tandis que dans ceux-ci le rotor tourne, il n'est soumis dans les régulateurs d'induction qu'à des déplacements angulaires limités et lents; le faible entrefer que les moteurs asynchrones doivent comporter ne constitue donc pas une difficulté dans le cas des régulateurs d'induction, machines dont la robustesse équivaut celle des transformateurs.

Notre but est d'indiquer successivement les propriétés caractéristiques des régulateurs d'induction pour courants polyphasés et pour courant monophasé; nous indiquerons ensuite les différents modes d'exécution et les divers emplois de ces appareils.

\section{1) RÉGULATEUR D'INDUCTION POUR COURANTS POLYPHASÉS}

a) Foncionnement. - Les enroulements de la partie induite F (fig. 1, en supposant le cas du triphasé) sont intercalés

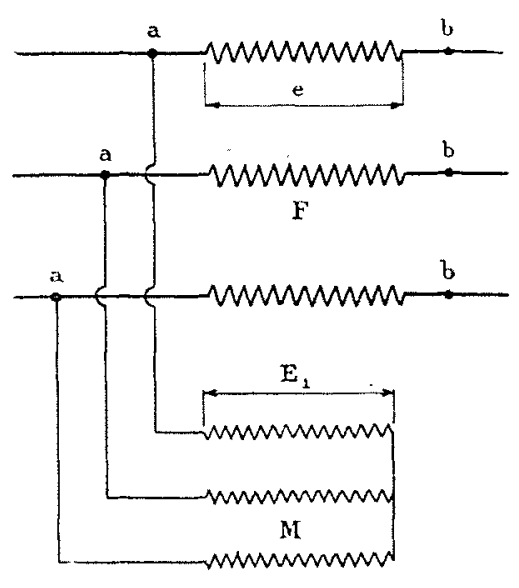

Fig. 1

en série avec le réseau à régler. Les enroulements de la partie inductrice $M$ sont branchés en dérivation sur les conducteurs du réseau et produisent un champ tournant qui induit dans chaque phase de la partie $F$ une tension e constante, mais qui, suivant la position relative des enroulements $\mathrm{M}$ et $\mathrm{F}$, se trouve plus ou moins déphasée par rapport à la tension d'alimentation $\mathrm{E}_{\mathbf{1}}$.

En effet, quand les enroulements de chaque phase du stator et du rotor se trouvent en face l'un de l'autre (fig. 2, où l'on n'a représenté pour plus de clarté que les enroulements d'une phase couvrant un pas polairc), lorsque le flux est maximum dans $M$, il l'est également dans F. La tension induite dans $F$ est donc en phase avec celle de $\mathrm{M}$ et par conséquent avec celle du réseau. Nous pouvons représenter cela graphiquement.

Désignons par $O A$ (fig. 3 ) la tension $E_{1}$ d'une phase du réseau ; en ajoutant $\mathrm{AB}_{1}=e$ en phase avec $\mathrm{OA}$, on obtient la tension d'une phase $O B_{1}=E_{2}$ du côté des bornes b (fig. 1) pour la position relative des enroulements $F$ et $M$ admise dans la fig. 2 .

(1) Extrait du Bulletin de la Société Alsacienne de Constructions Mécaniques.
Si on déplace la partie $M$ de façon à l'amener dans la position représentée par la fig. 4, la force électromotrice induite dans F est maximum à l'instant où elle est nulle en $M$, c'est-à-dire que la tension induite dans $\mathrm{F}$, toujours égale à $e$, est déphasée d'un

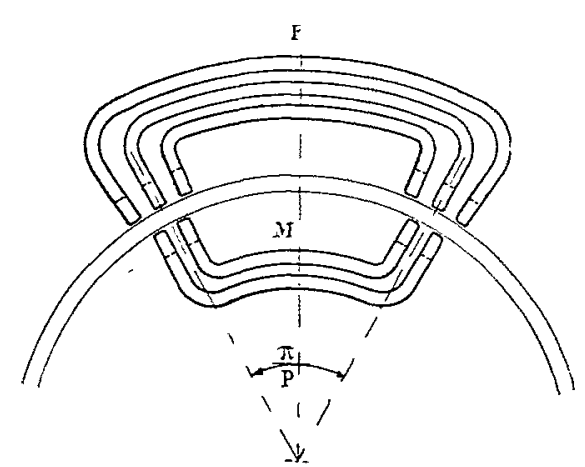

Fig. 2

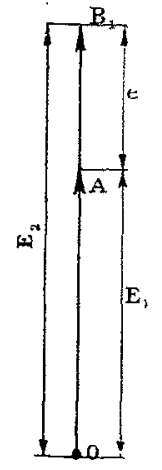

Fig. 3 quart de période par rapport à celle de M. Pour cette position, la tension par phase $\mathrm{E}_{2}$ du côté des bornes $b$ (fig. 1) est vectoriellement égale à $\mathrm{OB}$ (fig. 5). Si l'on continue encore à déplacer $\mathrm{M}$ par rapport à $\mathrm{F}$, on arrive, après un déplacement angulaire de $\frac{\pi}{p}(2 p=$ nombre de pôles de la machine), à une position telle que le maximum de tension positif dans $M$ coincide dans le temps avec le maximum de tension négatif de la force électro-

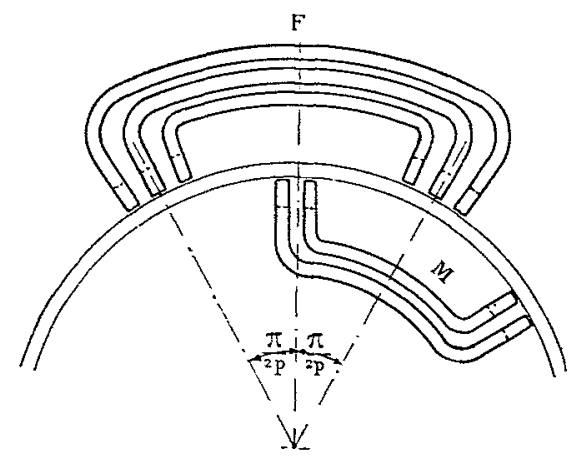

Fig. 4

motrice induite dans $\mathrm{F}$. La tension $e$ se retranche alors de $\mathrm{E}_{1}$ et on obtient du côté des bornes $b$ une tension représentée par $\mathrm{OB}_{2}=\mathrm{E}_{2}$ (fig. 6).

On peut donc réaliser, par le déplacement relatif de $F$ et de $\mathrm{M}$, n'importe quel déphasage entre $\mathrm{E}_{1}$ et $e$ et obtenir une tension résultante de valeur comprise entre $\mathrm{OB}_{1}=\mathrm{E}_{1}+e$ et $\mathrm{OB}_{2}=$ $\mathrm{E}_{1}$ - e (fig. 7). L'extrémité du vecteur $A B$ se déplace sur l'une, ou l'autre des demi-circonférences décrites sur $B_{1} B_{2}$ suivant que, partant de la position initiale de la fig. 2 , on déplace le rotor dans l'un ou l'autre sens; le déplacement angulaire total du rotor est alors égal à $\frac{2 \pi}{p}$ et équivaut au double pas polaire de la machine.

b) Courant inducteur. - Les régulateurs d'induction. sont construits de façon à ne permettre qu'un déplacement angulaire $\frac{\pi}{p}$ du rotor et correspondant à un simple pas polaire. Le point B. ne 
peut donc se déplacer que sur l'une ou l'autre des demi-circonférences $B_{1} B_{2} B_{2}$ et $B_{1} B^{\prime} B_{2}$, l'un et l'autre de ces réglages domnant la même variation de la tension. Nous allons montrer que si les deux modes de réglages sont équivalents au point de vue des réseaux d'utilisation et d'alimentation, ils conduisent cepen-

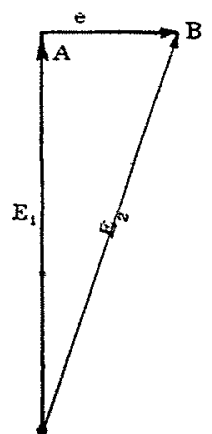

Fig. 5

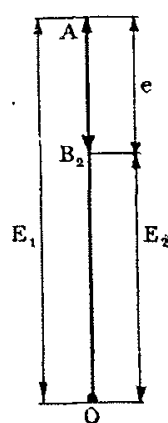

Fig. 6 dant pour le régulateur à des régimes de fonctionnement diffèrents, la différence étant d'autant plus grande que le courant magnétisant est plus important par rapport au courant débité sur le réseau d'utilisation. La fig. 8 représente la coupe schématique d'un régulateur d'induction à courants polyphasés supposé à 4 pôles, mais on n'a représenté que l'enroulement d'une phase supposé concentré dans une seule entaille par pôle, le bobinage

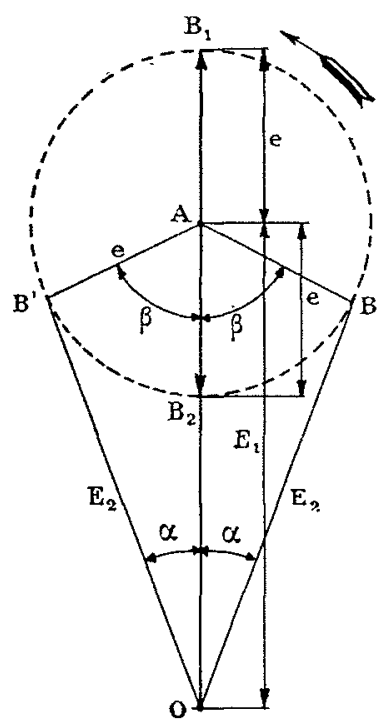

Fig. 7

étant du genre dit à pôles conséquents. Nous admettons que le champ tourne dans le sens des aiguilles d'une montre (sens de la flèche) et nous partons toujours comme position fondamentale de celle où les axes des enroulements $F$ et $M$ coïncident (position 1). D'après ce qui a été dit plus haut, les forces électromotrices induites dans chacun d'eux sont en phase ou en opposition suivant les sens d'enroulement relatifs et le vecteur OB représentant la tension résultante est soit en $\mathrm{OB}_{1}$, soit en $\mathrm{OB}_{2}$.

Pour préciser davantage, nous admettons que les sens d'enroulement sont les mêmes en partant des points a de la fig.1; en remarquant alors que, dans la fig. $7, \mathrm{OA}$ représente la tension d'alimentation aux bornes de l'enroulement M, cela veut dire que la force électromotrice induite opposée à la tension aux bornes est dirigée de $\mathrm{A}$ vers $\mathrm{O}$ (en négligeant les chutes ohmique et inductive) et par suite que $A B$ se place en $A_{2}$; la position admise (position 1) est celle de dévoltage maximum. Si on déplace le rotor dans le sens du champ tournant (en position 2 par exemple), l'enroulement $F^{*}$ va se trouver décalé en avance dans le champ par rapport à $M$ et sur la fig. 7 , la flèche indiquant le sens de rotation des vecteurs, le point $B_{2}$ se meut vers $B$. Si le déplacement du rotor a lieu au contraire en sens inverse de celui du champ (position 3) le mouvement du point $B_{2}$ s'effectue vers $B$ '. Dans le premier cas, la tension d'utilisation $O B$ est déphasée en retard d'un angle $\nsim$ sur la tension d'alimentation dans le second cas, elle est en avance.

A vide, l'enroulement $M$ est parcouru par le courant magnétisant; ce courant reste constant quelle que soit la position relative de F et de M. En charge, il faut que dans l'enroulement M s'établisse un courant tel que les ampère-tours résultant de ceux dus à $\mathrm{F}$ et à $\mathrm{M}$ restent égaux aux ampère-tours magnétisants. Tant que dans l'espace, les axes des enroulements $M$ et $F$ coïncident (position fondamentale), l'enroulement $M$ est done parcouru en charge par un courant produisant un nombre d'ampère.

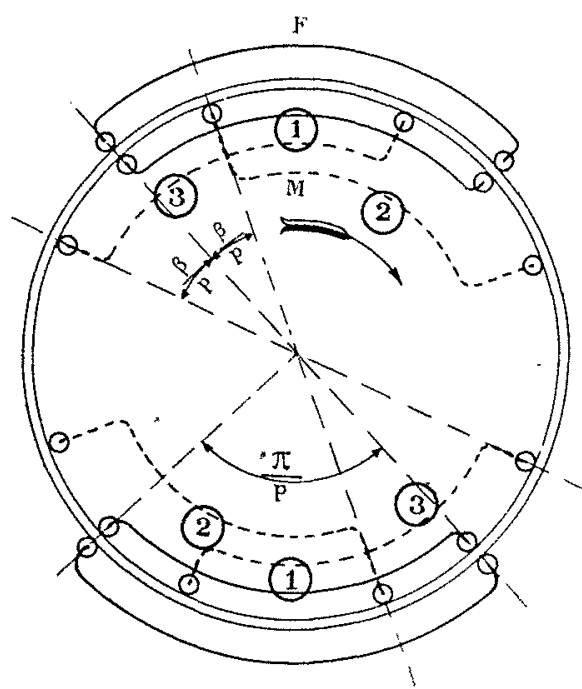

Fig. 8

tours égal à la résultante des ampère-tours magnétisants et des ampère-tours de l'enroulement $F$ pris en sens contraire. Mais si les axes des enroulements $M$ et $F$ ne coïncident pas dans l'espace, $\mathrm{F}$ étant, par exemple, décalé en avance d'un angle $\frac{\beta}{p}$ dans le champ par rapport à $M$ (position 2, fig. 8), pour obtenir l'équivalent des ampère-tours dus à $F$ par un courant circulant dans $M$, il faudrait imaginer dans cet enroulement un courant déphasé en reiard dans le temps sur le courant parcourant $F$ d'un angle $\beta$ correspondant au décalage $\frac{\beta}{p}$.

Le diagramme de la fig. 9 traduit ce fonctionnement pour le cas d'une charge purement active (courant $I_{u}$ dans l'enroulement $\mathrm{F}$ en phase avec $\mathrm{OB}$ ). Le courant magnétisant est porté en $\mathrm{OO}^{\prime} ; \mathrm{AO}$ est la force électromotrice induite dans $\mathrm{M}$, et $\mathrm{AB}$ la force électromotrice induite dans $\mathrm{F}$. Si $\mathrm{N}_{\mathrm{f}}$ et $\mathrm{N}_{\mathrm{m}}$ sont les nombres de conducteurs des enroulements $F$ et $M$, on sait que

$$
\frac{\mathrm{OA}}{\mathrm{AB}}=\frac{\mathrm{N}_{\mathrm{m}}}{\mathrm{N}_{\mathrm{f}}}
$$

Le courant $I_{u}$ dans l'enroulement $F$ équivaut, au point de vue des ampères-tours, à ún courant $I_{u}^{\prime}=I_{u} \frac{N_{f}}{N_{m}}$ dans l'enroulemenl $M$, représenté, à la même échelle que le courant magnétisant, par une longueur OK. D'après ce que nous avons dit ci-dessus, pour produire dans la machine un champ identique en grandeur et en phase dans l'espace à celui dû au passage du 
couranl $I_{\mathrm{u}}$ dans l'enroulement $\mathrm{F}$, il faudrait faire passer dans l'enroulement $\mathrm{M}$ un courant $\mathrm{I}_{\mathrm{u}}^{\prime}=0 \mathrm{~K}$, déphasé dans le temps d'un angle $\beta$ en arrière par rapport à OK. Pour la charge et la position considérées, le courant $I_{m}$ dans $M$ est la résultante de $O O$ ' et dé $O$ ' $B_{1}$ égal et directement opposé à OK'.

Tandis que pour la position fondamentale, le courant rotorique est $\mathrm{OH}$, pour la position considérée il n'est plus que $\mathrm{OB}_{1}$. $\Lambda$ partir de la position de dévoltage maximum, le mouvement du rotor s'effectuant dans le sens du champ tournant et variant de $O$ à $\frac{\pi}{2}$ pour la machine à 4 pôles considérée el plus généralement dè $O$ a $\frac{\pi}{p}$ pour une machine à $2 p$ pôles, le point $\mathrm{B}$ se déplace de $B_{2}$ à $B_{3}$ sur la demi-circonférence tracée en trait plein $B_{2} B B_{3}$ et pour une charge purement active l'extrémilé $B_{1} d u$ vecteur du courant rotorique se déplace de $\mathrm{H}$ à $\mathrm{H}^{\prime}$ sur la demi-

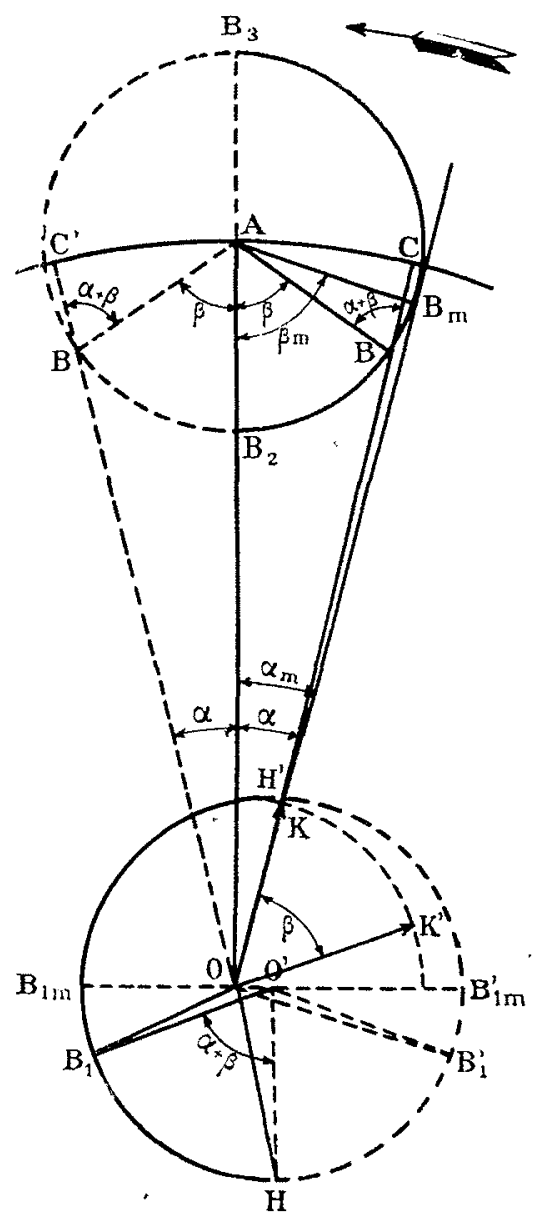

Fig. 9

circonférence $\mathrm{H} \mathrm{B}_{1} \mathrm{H}^{\prime}$ de centre $\mathrm{O}^{\prime}$ et de rayon $\mathrm{I}_{\mathrm{u}}^{\prime}=\mathrm{OK}=\mathrm{O}^{\prime} \mathrm{H}$. Le courant rotorique passe par un minimum $\mathrm{OB}_{1 \mathrm{~m}}$ pour une valeur $\beta_{\mathrm{m}}$ de $\beta$ légèrement inférieure à $\frac{\pi}{2}$, la valeur $\beta_{\mathrm{m}}$ correspondante étant telle que $\beta_{\mathrm{m}}+x_{\mathrm{m}}=\frac{\pi}{2}$, avec $x_{\mathrm{m}}=\arcsin \frac{\mathrm{N}_{\mathrm{f}}}{\mathrm{N}_{\mathrm{m}}}$.

Il est clair maintenant que si, au lieu de supposer le mouvement du rotor, à partir de la position fondamentale, effectué dans le sens du champ tournant, nous le supposons effectué dans le sens inverse, le point $B$ se déplace alors sur la demi-circonférence tracée en pointillé $B_{2} B^{\prime} B_{3}$, la tension d'utilisation varie encore de la valeur minimum $\mathrm{OB}_{2}$ à la valeur maximum $O B_{3}$ et l'extrémité $B_{1}$ du courant rotorique se déplace sur la demi-circonférence $\mathrm{HB}_{1}{ }_{1} \mathrm{H}^{\prime}$ de centre $\mathrm{O}^{\prime}$ tracée également en pointillé. Le courant rotorique passe alors pạr un maximum
$O B_{1 m}^{\prime}$ pour lquel $\beta_{m}=\beta_{m}$. On se rend facilement compte que pour le déplacement $\frac{\beta_{m}}{p}$ du rotor, la tension d'utilisation $O B$ est sensiblement égale à la tension d'alimentation OA, c'està-dire que les fonctionnements particuliers signalés, à courant rotorique maximum ou minimum, correspondent à peu près à la position de survoltage-dévoltage nul pour une charge active.

Si le courant de charge n'est pas purement actif, mais déphasé, par exemple en arrière d'un angle ou sur la tension d'utilisation, dans le premier fonctionnement envisagé (déplacement du rotor dans le sens du champ tournant), le courant part d'une valeur d'autant plus voisine du minimum que le déphasage est plus grand, le vecteur $O^{\prime} B_{1}$ faisant avec $O^{\prime} H$ un angle $\left(x+\beta+\varphi_{u}\right)$ déjà égal à î pour la position initiale admise. Le courant $I_{m}$ passe par son minimum pour un faible déplacement et prend des valeurs toujours croissantes avec le survoltage. Pour le déplacement du rotor en sens inverse du champ tournant, le vecteur du courant inducteur $\mathrm{O}^{\prime} \mathrm{B}_{1}$ fait avec $\mathrm{O}^{\prime} \mathrm{H}$ un angle $\left(\alpha+\beta-\rho_{\mathrm{u}}\right)$ déjà égal à - qu pour la position initiale admise; il part de la même valeur que ci-dessus, croît d'abord avec la tension pour passer par son maximum avant que cette dernière atteigne sa plus grande valeur et décroît ensuite. Pour une charge purement réactive, quel que soit le sens du déplacement du rotor, le courant inducteur part de sa valeur minimum pour $\frac{\beta}{p}=0$ et atteint sa valeur maximum pour $\frac{\beta}{p}=\frac{\pi}{p}$.

Nous devons remarquer d'ailleurs que, pour le premier mode de fonctionnement envisagé, le courant $\mathrm{OB}_{1 \mathrm{~m}}$ est en avance de $\frac{\pi}{2}$ sur la tension d'alimentation et on sait qu'à un tel fonctionnement correspond une force électromotrice induite supérieure à la tension aux bornes, c'est-à-dire une augmentation du flux et par suite des pertes dans le fer de la machine. Il en résulte que si les pertes dans le cuivre inducteur diminuent, les pertes dans le fer augmentent. Il est toutefois généralement recommandable d'adopter le fonctionnement à courant rotorique minimum, le rotor étant la partie la plus difficile à refroidir et la valeur moyenne des pertes totales dans toute l'étendue du réglage probablement un peu plus faible.

Signalons encore une particularité du fonctionnement au courant inducteur minimum. Si le courant du réseau d'utilisation a une valeur telle que le vecteur représentatif OK (fig. 9) est égal à OO', le courant inducteur minimum correspondant est nul, c'est-à-dire qu'on peut à ce moment ouvrir le circuit inducteur 'sans apporter aucun trouble au fonctionnement. Ceci est tout à fait normal, car dans ce fonctionnement, le courant traversant l'enroulement $F$, dirigé suivant $\mathrm{OB}_{\mathrm{m}}$, est égal au cou-

rant magnétisant; la tension de réglage $A_{\mathrm{m}}$ est déphasée de $\frac{\pi}{2}$ en arrière par rapport $\mathrm{OB}_{\mathrm{m}}$. Tout se passe donc exactement comme si, l'enroulement inducteur $M$ étant ouvert, l'enroulement induit $F$ était intercalé comme une self-induction pure en série dans le réseau d'utilisation. Le flux uniquement dû aux ampère-tours de l'enroulement $F$ est égal au flux normal; la force électromotrice induite par ce flux et mesurée aux bornes de $M$ est égale à la tension du réseau d'alimentation et en phase avec cette tension de sorte qu'on peut relier $\mathrm{M}$ au réseau sans qu'aucun courant prenne naissance.

Le clavetage du secteur denté produisant le déplacement du rotor étant réalisé une fois pour toutes par le constructeur et fixant le sens de ce déplacement, ce que nous avons dit précédemment permet à l'exploitant de se rendre facilement compte 
du changement de conmexions nécessaire pour passer d'un mode de fonctionnement à l'autre. Il suffit, en effet, soit de croiser deux fils à l'alimentation, ce qui inverse le sens du champ lournant mais conserve les positions de survoltage et de dévoltage maximum pour les mèmes positions de la partie mobile, soit d'inverser toutes les phases de l'un des enroulements par rapport à l'autre (on choisira de préférence l'enroulement induit puisque toutes ses extrémités sont accessibles), auquel cas lc sens du champ tournant subsiste, mais la position du secteur denté qui correspondait, par exemple, au dévoltage maximum sera alors celle du survoltage maximum et vice-versa.

c) Couple. - On conçoit facilement qu'en charge le rotor du régulateur d'induction est soumis à un couple de rotation électromagnétique. Comme pour le rotor d'un moteur asynchrone, ce couple est proportionnel à la composante du courant rotoricque en quadrature avec $00^{\prime}$. Si cette composante est dirigée suivant $O A$, le rotor absorbe de la puissance qu'.l transmet au stator et le couple tend à entraîner le rotor en sens inverse du champ tournant. Si la composante est dirigée suivant $\mathrm{AO}$, le rotor est au contraire soumis à un couple dans le sens du champ comme dans un moteur asynchrone, la puissance étant transmise du stator au rotor et renvoyée par celui-ci au réseau. Pour une charge active, au voisinage de la position moyenne de réglage, le couple s'exerçant sur le rotor s'annule. Il ne faut donc pas craindre que le rotor d'un régulateur d'induction, mécaniquement libre, se mette à tourner et tende à atteindre la vitesse de synchronisme correspondant au nombre de pôles de la machine. Dans ce cas, le rotor vient simplement occuper la position pour laquelle le couple est nul et de part et d'autre de laquelle il est soumis à des couples de sens inverses. Pour que le couple soit toujours de même sens, il faudrait que la tension induite dans la partie $F$ fût plus grande que dans la partie inductrice, c'est-àdire $\mathrm{AB}>\mathrm{OA}$, le lieu du courant rotorique est alors limité à une zone de la demi-circonférence $B_{1 m} H^{\prime} B_{1 m}^{\prime}$ de centre $O^{\prime}$ et le couple toujours en sens inverse du sens du champ tournant. Notons également en passant qu'un régulateur d'induction pour courants polyphasés dans lequel on aurait $A B>0 A$, ou $N_{f}>N_{m}$ ne permet cependant pas d'obtenir une tension d'utilisation rigoureusement nulle (nous verrons plus loin que ceci est possible avec le régulateur d'induction monophasé).

d) Puissances, colrants et tensions utiles et absorbés. Indiquons maintenant une construction simple permettant d'obtenir facilement les tensions et les courants dans les différents circuits. Comme dans la fig. 9, on a tracé sur la fig. 10 la circonférence de centre $A$ et telle que $\frac{A B}{\mathrm{OA}}=\frac{N_{\mathrm{f}}}{N_{m}}$. Pour une position du rotor définie par l'angle $\beta$, la tension d'utilisation est $O B$; en choisissant l'échelle des courants telle que OA représente le courant d'utilisation, le courant équivalent dans l'enroulement inducteur $M$ est représenté par une longueur $O A \frac{N_{f}}{N_{m}}=A B$. Si du point $O$ comme centre, on trace les circonférences de rayons $O A$ et $O B$, la première coupe $O B$ en $C$ et la seconde coupe $O A$ en $D$ : On se rend facilement compte que l'angle $\widehat{O C D}$ est aussi égal à $\beta$ et l'angle $\widehat{\mathrm{ADC}}$ égal à $(x+\beta) ; \mathrm{CD}$ représente en grandeur et en phase le courant dans l'enroulement $M$ pour une charge purement active, abstraction faite du courant magnétisant. En portant celui-ci en DI, on a en CI le courant réel dans l'inducteur $\mathrm{M}$ et $\mathrm{OC}$ étant le courant dans l'induit $\mathrm{F}$, on a en OI le courant total absorbé au réseau. Pour un déplacement du rotor égal et de sens inverse à celui considéré, on aurait respectivement $\mathrm{OB}^{\prime}=$ tension d'utilisation, $\mathrm{C}^{\prime} \mathrm{D}=$ courant dans l'induc- leur (compte non tenu du courant magnétisanl), C'I = couranl réel dans l'inducteur et $O I=$ courant tolal absorbé au réscau. Si la charge correspond à un facteur de puissance cos pu, on trouve, comme indiqué sur la fig. 10, les points correspondants désignés par les mêmes lęltres caractérisées par l'indice q. Celle construction montre que pour une mème charge, le courant absorbé au réseau $O I$ ou $\mathrm{OI}_{\text {o }}$ est le mème en grandeur el en phase, quel que soil le déplacement du rolor réalisé par rapport au sens du champ tournant.

Comme nous n'avons introduit jusqu'ici dans la construction ni les chutes ohmiques ni les chutes inductives, on déduil lacilement du diagramme que la puissance aclive absorbée est

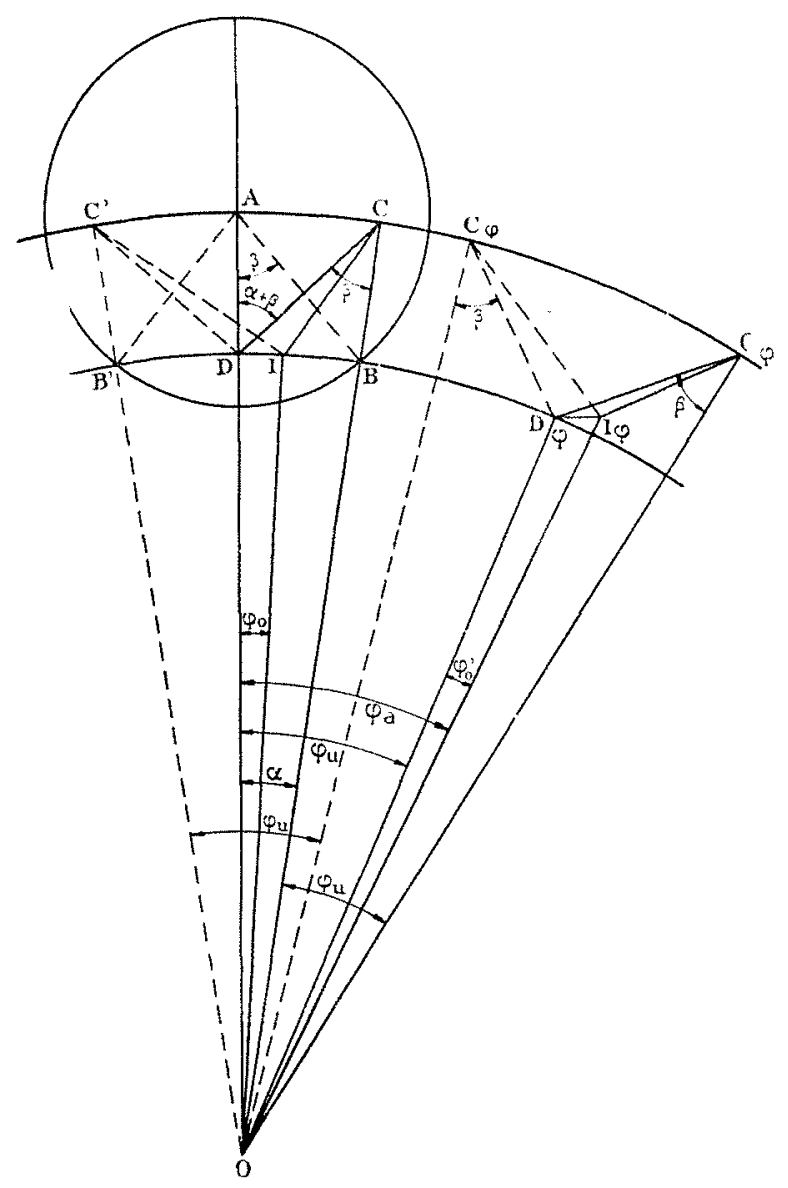

Fig. 10

égale à la puissance active à l'utilisation et que la puissance réactive absorbée est égale à la puissance utile réactive majorée de celle nécessaire à la magnétisation du régulateur d'induction Iui-même.

L'introduction rigoureuse des différentes chutes ohmiques et inductives dans le diagramme est assez compliquée et ne s'effectue généralement qu'après quelques làtonnements, par approximations successives. On comprend facilement que leur influence se traduise généralement par une légère diminulion de la tension d'utilisation $\mathrm{OB}$, par rapport à sa valeur théorique et par une augmentation du courant absorbé à l'alimentation en même temps que par un plus grand déphasage de ce courant. La puissance active absorbée est supérieure à la puissance active utile, de la valeur des pertes dans le régulateur. La puissance réactive absorbée diffère de la puissance réactive utile non seulement de la valeur nécessaire à l'aimanlation, mais aussi de celle correspondant aux fuiles des enroulements.

Les pertes d'un régulateur d'induction sont comprises entre relles d'un transformateur et celles d'un moteur asynchrone (frottements et ventilation non compris) de même puissance 
interne apparenle ( $\mathrm{I}$ par phase). En admettant que les pertes sonl cgales à cinq pour cent de la puissance interne, si l'appareil règle de $10 \%$ le tension d'un réseau, les pertes ne représentent donc que $05 \%$ de la puissance traversante et le rendement d'un tel appareil, délini par le quotient de la puissance côté utilisation et de la puissance côlé alimentation serail donc de $99,5 \%$. Pour éviler toute confusion, nous jugeons préférable d'indiquer pour ces appareils la valeur des pertes à vide et en charge.

e) Régulatelk d'induction dolble. Le régulateur d'induction polyphasé que nous renons de décrire provoque donc un ecart entre les phases des lensions d'ulilisation et d'alimentation. Cet écarl, quelquefois utilisé, n'entraîne généralement pas d'inconvénients; lontefois dans le cas de réseaux bouclés entre cux à l'amont et à l'aval d'un régulateur d'induction, il en résulterail des courants de circulation inadmissibles. On emploie alors pour le réglage de la tension deux régulateurs d'induction, dont les enroulements $F_{1}$ et $F_{2}$ sont montés en série et les enroulements $M_{1}$ et $M_{2}$ en parallèle comme indiqué par le schéma de la fig. 11, en supposant les régulateurs identiquement bobinés.

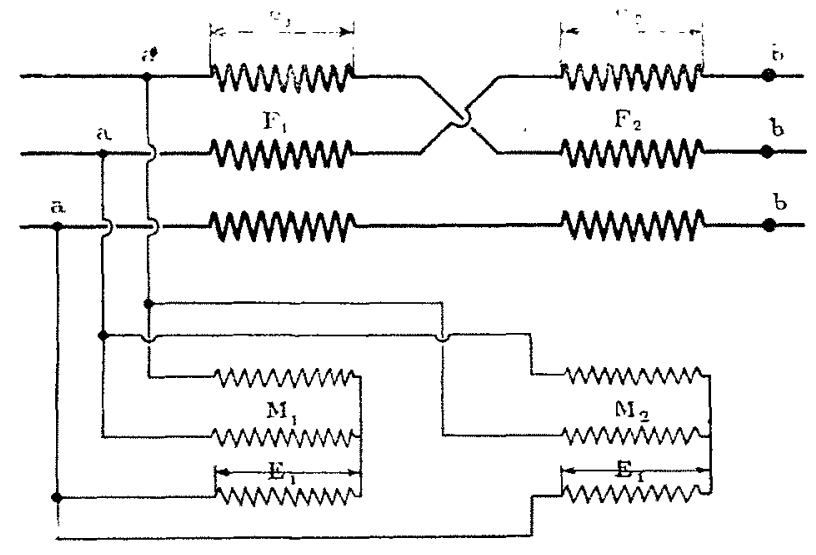

Fig. 11

Les sens de rotation des cloamps sont inverses l'un de l'autre dans les deux régulateurs. Dans la position de dévoltage maximum les tensions $e_{1}$ et $e_{2}$ égales entre elles, s'ajoutent arithmétiquement et la tension d'utilisation est égale à $E_{1}-\left(e_{1}+e_{2}\right)=$ $\mathrm{E}-2 e$, c'est-à-dire que pour un réglage total égal à \pm 2 e, chacun des régulateurs d'induction est à dimensionner pour une tension moitié $c_{1}=c_{2}=e$. Partant de la position de dévoltage maximum, pour un déplacement angulaire de mème sens $\frac{\pi}{p}$ des rotors, supposés mécaniquement liés entre eux, l'un des rotors se déplace dans le sens du champ tournant et l'autre en sens inverse, de sorte que la tension $e_{1}$ se place par exemple en $\mathrm{AB}$ (fig. 12) et la tension $e_{2}$ se place en $\mathrm{AB}^{\prime}$; la tension d'utilisation résultante $O A$ ' reste toujours dirigée suivant $O A$, c'està-dire en phase avec la tension d'alimentation. La phase du courant d'ulilisation I correspondant à arc cos ₹u est toujours à compter à partir de $O A$ et les courants rotoriques $O B_{1}$ et $O B_{1}$ dans chacun des enroulements $M_{1}$ et $M_{2}$ s'obtiennent en portant à partir de $O^{\prime}$ les vecteurs $O^{\prime} B_{1}$ et $O^{\prime} B^{\prime}{ }_{1}$ faisant respectivement avec $\mathrm{O}^{\prime} \mathrm{H}$ des angles $\left(\hat{\beta}+\sigma_{1}\right)$ et $\left(\hat{\beta}-\tau_{\mathrm{i}}\right)$. Dans la position initiale, les courants rotoriques des régulateurs sont égaux entre eux et en phase et représentés par $\mathrm{O}^{\prime} \mathrm{H}_{1}$. Pour la plupart des positions de réglage, les courants rotoriques diffèrent en grandeur et en phase, ce qui nécessite la mise en parallèle des enroulements $M_{1}$ et $M_{2}$ et exclut l'emploi d'un montage avec ces enroulements en série comme pour $F_{1}$ et $F_{2}$.
On peut obtenir une tension de réglage présentant par rapport $\dot{a} O \mathrm{~A}$ un déphasage quelconque et constant dans toute l'amplitude du réglage. En particulier, si on inverse la tension $e_{2}$ par exemple, elle prend la direction $A B^{\prime \prime}$ et la tension de réglage reste toujours dirigée suivant $\mathrm{AA}$ ", en quadrature avec OA.

Dans le cas des appareils utilisés suivant le montage de la fig. 11, surtout si la puissance n'est pas très grande, on monte généralement les parties mobiles des deux régulateurs d'induction sur le même arbre (fig. 13), et on caractérise l'appareil ainsi obtenu par la dénomination de régulateur d'induction double, le régulateur d'induction simple étant celui que nous avons d'abord décrit. Dans un tel appareil, le couple s'exerçant

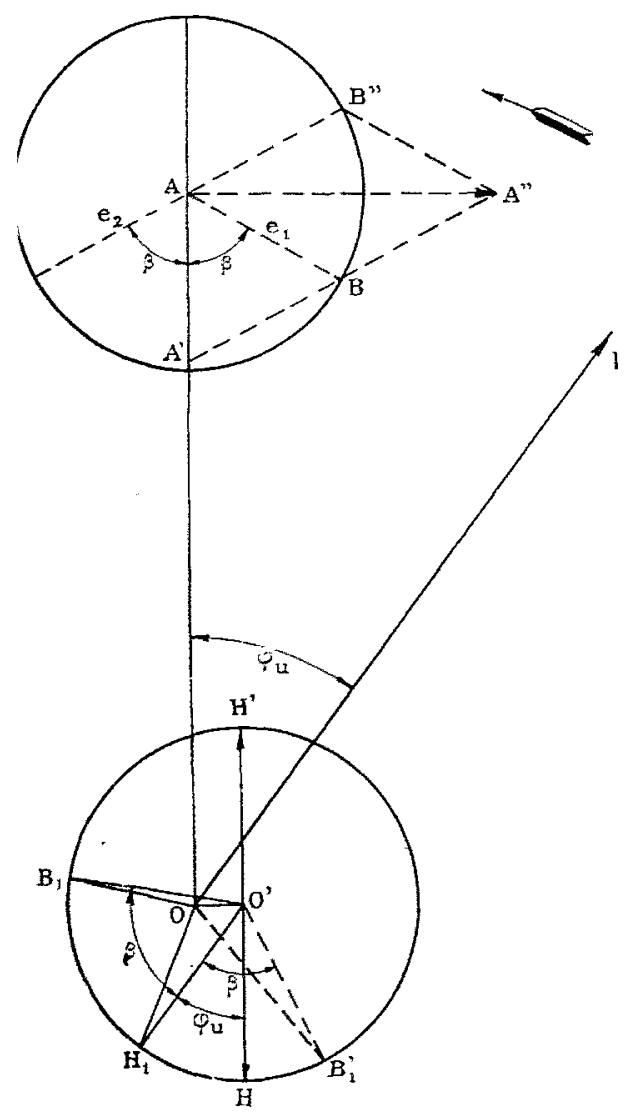

Fig. 12

sur la partie mobile est la somme des ccuples s'exercant sur chacune des moitiés. Nous avons montré que chacun de ces couples est défini en grandeur et en sens, par rapport au sens du champ tournant, par la projection du courant rotorique suivant O'H. Le rapport du couple total résultant au couple maximum de chaque demi-régulateur est donc proportionnel à :

$$
\cos \left(\beta-\varphi_{u}\right)-\cos \left(\beta+\sigma_{u}\right)=2 \sin \beta \sin \varphi_{u}
$$

Le couple total dépend donc du facteur de puissance du circuit d'utilisation; pour une charge purement active $\left(p_{u}=0\right)$, il est toujours nul et ceci constitue un autre avantage du régulateur d'induction double; la partie mobile peut être facilement déplacée quelle que soit sa position. Cet avantage s'atténue au fur et à mesure que le facteur de puissance $\cos \rho_{\mathrm{u}}$ diminue. Pour $\cos q_{\mathrm{u}}=0,8$, le couple atteint 1,2 fois le couple maximum d'un demi-régulateur; il correspond à la position pour laquelle $\beta=\frac{\pi}{2}$; cette position est équidistante des positions extrêmes de réglage pour lesquelles le couple est nul dans tous les cas.

Le coût élevé du régulateur d'induction double en réduit l'emploi et on utilise surtout le régulateur d'induction simple, 
malgré ses légers inconvénients. En raison de la grande démultiplication entre le mouvement du rotor et celui de l'arbre de commande, on équilibre facilement le couple électromagnétique de l'appareil et on réalise le réglage en ramenant l'effort à une valeur raisonnable sans que le temps nécessaire pour opérer cas de la fig. 4, les ampère-tours de $\mathrm{F}$ ne peuvent plus être neutralisés par M. Pour cette position, l'enroulement $F$ représenterait donc une self-induction considérable montée en série sur le réseau, provoquant une chute de tension inductive importante et entraînant un échauffement exagéré du fer. Aussi

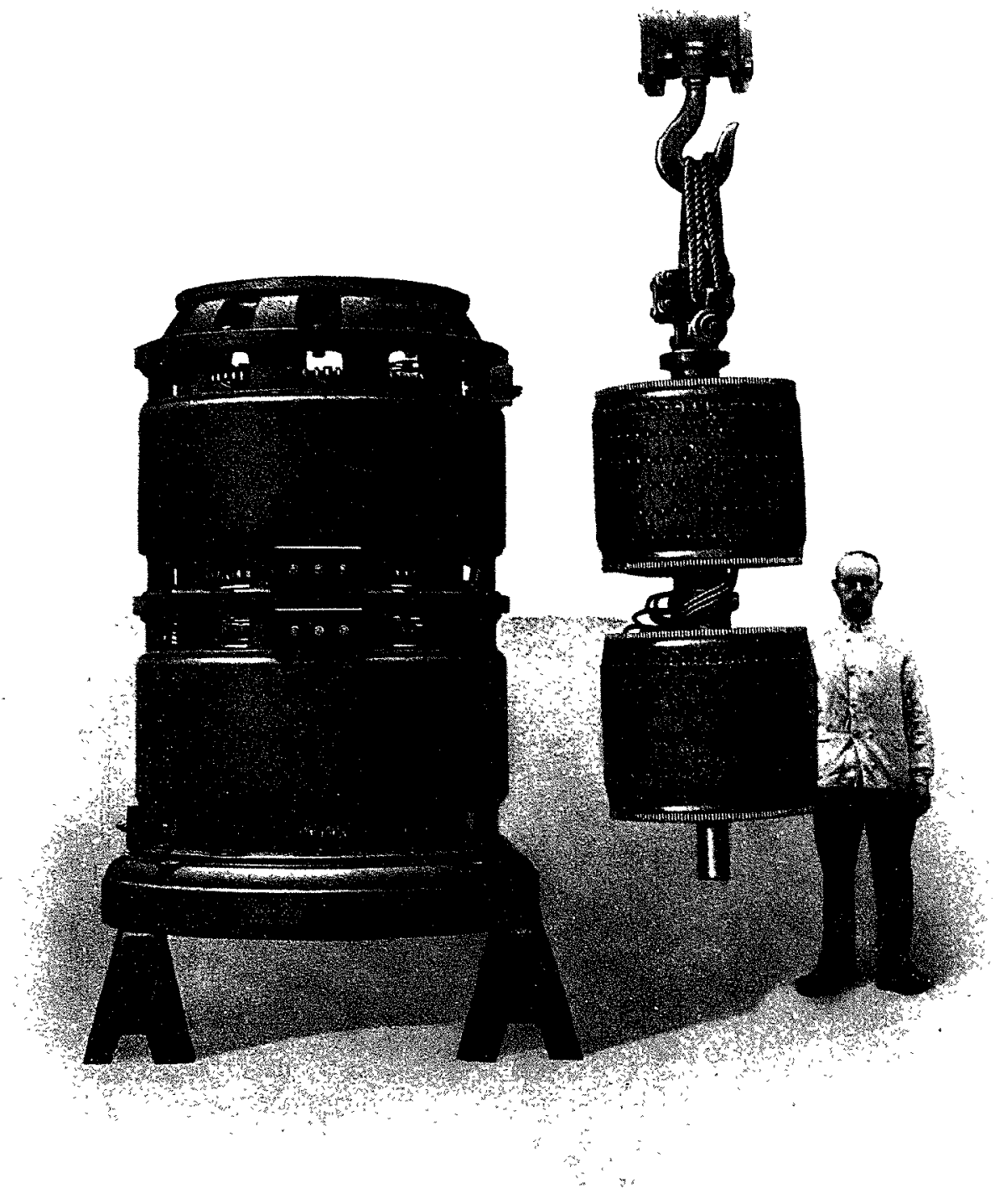

Fig. 13. - Régulateur d'induction double

le déplacement total du rotor excède une minute. Dans le même but, on adopte pour les enroulements du régulateur un nombre de pôles $2 \mathrm{p}$ assez faible. Les machines à 2 pôles présentant certaines difficultés de construction, on n'emploie ce nombre de pôles que pour les puissancés élevées; dans tous les autres cas, on fait généralement les machines à 4 pôles. Quant au déphasage introduit par l'appareil, sauf pour les réseaux bouclés, il n'a pas d'influence dans la plupart des cas, même dans celui où des commutatrices alimentées par le même réseau alternatif fonctionnent également en parallèle du côté continu.

\section{II) RÉGULATEUR D'INDUCTION POUR COURANT MONOPHASE}

a) Fonctionnement. - En se reportant aux fig. 2 et 4, om conçoit facilement le fonctionnement d'un régulateur d'induction sur réseau à courant alternatif monophasé. Dans le cas de la fig. 2, les ampère-tours dus à $F$ correspondant au passage du courant à travers le régulateur sont équilibrés par un nombre d'ampère-tours équivalents dus à l'enroulement $M$, mais dans lẹ est-on obligé, dans les régulateurs d'inducti-n à courant monophasé, de disposer sur la partie inductrice, en outre de l'enroulement $M$, un enroulement amortisseur M' (fig. 14), en

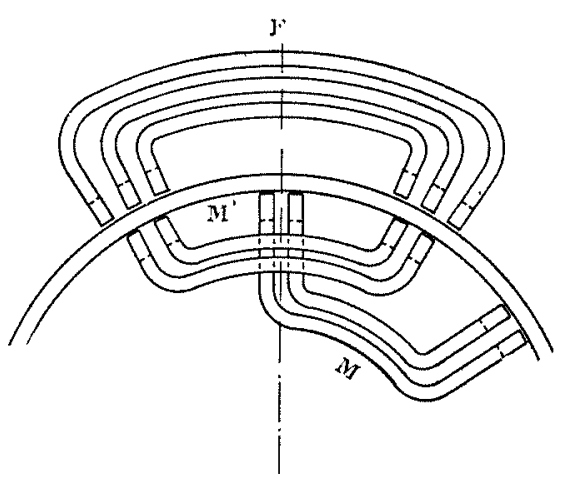

Fig. 14

quadrature avec le premier. Cet enroulement M' est simplement court-circuité sur lui-même; le courant qui le parcourt varie 
suivant la position relative des enroulements $F$ et $M$ '. Ce courant est maximum pour la position correspondant aux fig. 4 et 14 ; il est nul pour la position correspondant à la fig. 2. Dans ces conditions, le flux que tendrait à produire le champ dû à $F$ est sensiblement réduit au seul flux de fuile par l'action soil séparée, soil simultanée des enroulements $M$ et $M$ '. La valeur de la machine peul être caractérisée par la variation $\Delta e_{\mathrm{cc}}$ de la tension aux bornes $e_{\mathrm{cc}}$ à appliquer à l'enroulement $\mathrm{F}$ lorsque, $\mathrm{M}$ et $M$ ' étant courl-circuités, on déplace la partie mobile d'un demipas polaire en maintenant constant le courant $I_{c c}$ dans $F$. En prenant, par exemple, une machine à enroulement triphasé normal donl on ulilise deux phases pour constituer l'enroulement $\mathrm{M}$ et la troisième pour l'enroulement $\mathrm{M}$ ', si l'enroulement $\mathrm{F}$ est comme l'enroulement $M$ réparti sur les 213 du pas poliire, il est évident que dans la position de survoltage (ou dévoltage) maximum, la valeur de $e_{\text {cc }}$ est très faible, les enroulements $M$ et $F$ présentant le long de l'entrefer la même répartition des conducteurs et les champs dus à ceux-:i pouvant s'équilibrer parfaitement. Pour cette position, le courant dans $M$ ' est rigoureusement nul. Dans la position moyenne, si les ondes fondamentales des champs produits par F et M' s'annulent encore, il subsiste cependant des champs résiduels dus à ce que les conducleurs de ces enroulements ne sont pas répartis sur la même fraction du pas polaire. Les flux produits par ces champs restent en quadrature avec $M$, et n'induisent aucune tension dans cet enroulement; par contre, pour écquilibrer les tensions induites par ces flux dans l'enroulement $F$, il est nécessaire de lui appliquer une tension $\left(e_{\mathrm{cé}}+\Delta \dot{e}_{\mathrm{cc}}\right)$ plus grande de $\lrcorner e_{\mathrm{cc}}$ que dans le premier cas. $\lrcorner e_{\mathrm{cc}}$ peut atteindre une valeur double de celle de $e_{\mathrm{ce}}$.

En marche normale, ces flux subsistent et entrainent une déformation de la tension induite dans $\mathrm{F}$ par rapport à la sinusoïde et par suite de la tension d'utilisation. Si cette déformation est sans conséquence pour certaines applications, elle peut cependant ètre inadmissible dans d'autres cas. On l'atténue en donnant à M' presque la même importance qu'à $M$, ce qui entraîne une moins bonne utilisation de la machine et la renchérit. La Société Alsacienne a réalisé des régulateurs d'induction monophasés dans lesquels les enroulements $M$ et $M^{\prime}$, également dimensionnés et convenablement disposés, assurent une déformation pratiquement négligeable de la tension.

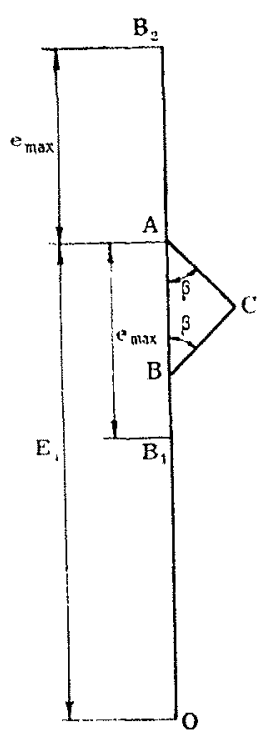

Fig. 15
Dans le régulateur d'induction monophasé, supposé convenablement amorti, conformément à ce que nous venons de dire, le flux total résultant pulse coaxialement à l'enroulement $\mathrm{M}$, mais sa valeur reste constante. C'est la fraction de ce flux traversant l'enroulement $\mathrm{F}$ qui détermine la tension induite, la phase de celle-ci est constante, sa grandeur seule varie. C'est donc l'inverse de ce qui se passe avec les courants polyphasés. L'appareil ajoute une tension $\pm e$ variable en valeur absolue entre zéro et un maximum correspondant à la position de la fig. 2 ; il permet donc d'obtenir en partant d'une tension OA (fig. 15) une tension d'utilisation de même phase que $\mathrm{OA}$ et comprise entre $\mathrm{OB}_{1}$ et $\mathrm{OB}_{2}$. On peut également se représenter le fonctionnement du régulateur d'induction monophasé en imaginant le champ pulsatoire comme formé, suivant un théorème bien connu, de deux champs tournants en sens inverses, d'amplitudes égales chacune à la moitié de celle dụ champ pulsatoire. Chacunn dẹ ces flux induit des tensions $\mathrm{AC}=\mathrm{CB}=\frac{e_{\max }}{2}$ dans l'enroulement $\mathrm{F}$ et la tension résultante, pour un déplacement $\frac{\beta}{p}$ de la partie mobile s'obtient, comme pour le régulateur d'induction double, en construisant le triangle isocèle ACB ayant à sa base des angles égaux à ß. Cette tension résultante $O B$ est évidemment égale à

$$
E_{1}-e_{\max } \cos \beta
$$

b) Couple. - Le couple électro-magnétique à vaincre pour effectuer le déplacement de la partie mobile dépend de la position relative des enroulements inducteur et induit et du facteur de puissance, comme pour les régulateurs d'induction polyphasés simple et double. Il est toujours nul aux positions de survoltage ou de dévoltage maximum; il est maximum dans la position moyenne de réglage si la charge- est entièrement active. Dans cette même position, pour une charge entièrement réactive, le couple est pulsatoire à une fréquence double de celle de la tension d'alimentation et s'exerce également, tantôt dans un sens, tantôt dans l'autre, de sorte que sa valeur moyenne est encore nulle. Pour des positions intermédiaires, le couple conserve son caractère pulsatoire, mais s'exerce plus longtemps et plus fortement dans un sens; sa valeur moyenne n'est plus nulle.

\section{III) DIFFERRENTS TYPES DE RÉGULATEURS D'INDUCTION}

de la Société Alsacienne de Constructions Mécaniques

Nous avons déjà dit que le régulateur d'induction est de construction semblable à celle d'un moteur asynchrone, mais le déplacement de la partie mobile, d'ailleurs intermittent, ne peut être utilisé pour assurer par ventilation le refroidissement de la machine. On emploie donc les mêmes moyens et les mèmes agents de refroidissement (air et huile) que pour les transformateurs statiques; le refroidissement des enroulements, en partie noyés dans le fer, étant plus difficile que dans les transformateurs, il faut déjà faire appel à ces agents pour des machines de puissance plüs faible que dans le cas des transformateurs.

Appareils dANS, L'AIr. - Pour les petites puissances (jusqu'à $10 \mathrm{kVA}$ au maximum), les régulateurs sont exécutés par la

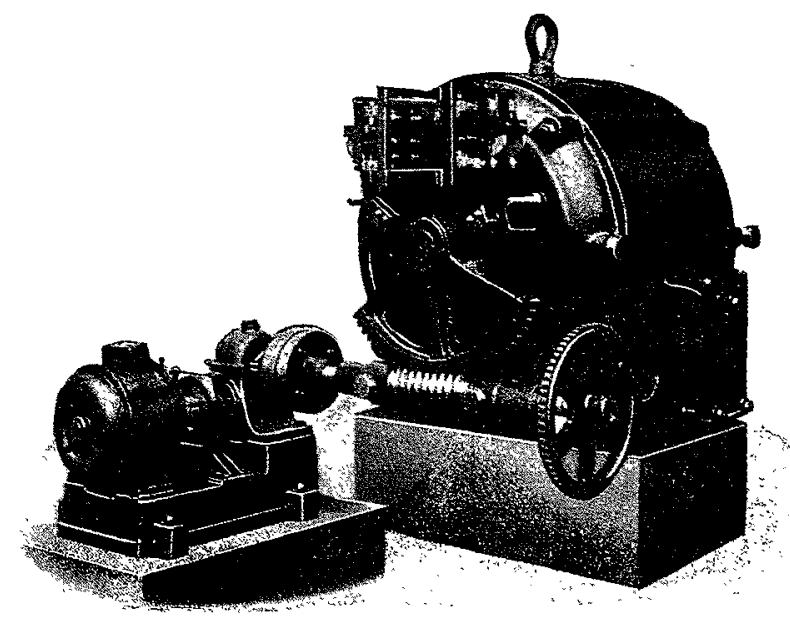

Fig. 16. - Régulateur d'induction triphasé ou monophasé type $\mathrm{ZV}$

$43 \mathrm{kVA}-0$ à 600 volts - 50 périodes

Société Alsacienne avec les mêmes organes mécaniques qu'un moteur asynchrone et se refroidissent naturellement. 
Pour les moyennes puissances (jusqu'à 150 à $200 \mathrm{kVA}$ ), les régulateurs sont encore à axe horizontal (fig. 16 et 17), mais un ventilateur, entraîné par un petit moteur auxiliaire visible sur le côté droit de la fig. 17, provoque un courant d'air à travers

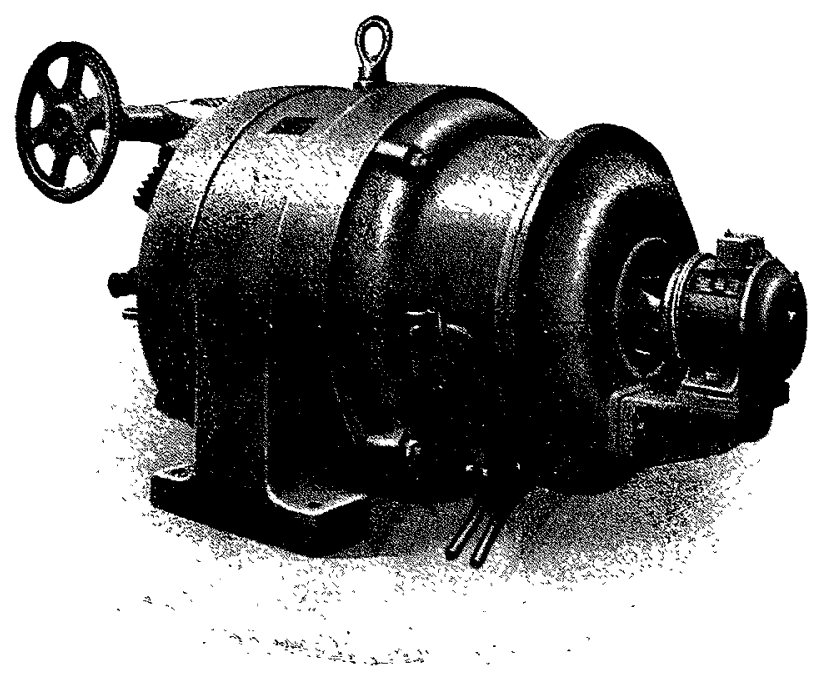

Fig. 17. - Régulateur d'induction triphasé ou monophasé type ZV

$35 \mathrm{kVA}-0$ à 200 volts - 50 périodes

des canaux axiaux convenablement disposés pour le refroidissement de toutes les parties du fer et des enroulements. Ce type de régulateur convient pour les tensions de 3000 et mème 5000 volts, surtout s'il doit ètre placé dans une salle propre et sèche.

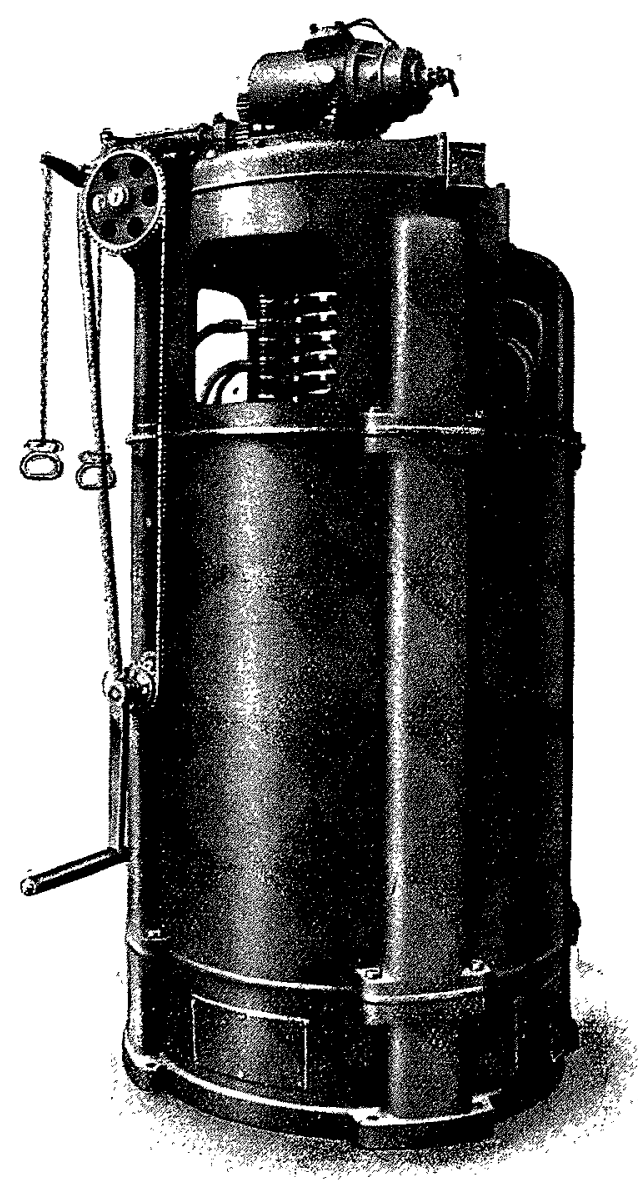

Fig. 18. - Régulateur d'induction hexaphasé type ZYV à refroidissement artificiel par ventilation. $930 \mathrm{KVA}: \pm 54$ volts par phase -2900 ampères, $412 / 3-50$ périodes, à commande électrique à distance et à commande à main pour le réglage de la tension d'une commutatrice de $3000 \mathrm{~kW}$.
Pour les grandes puissances, la Société Alsacienne utilise la disposition à axe vertical (fig. 18). Le régulateur est placé au-dessus d'une chambre dans laquelle on crée une pression d'air à l'aide d'un ventilateur; cette pression provoque dans tout l'appareil, a travers des canaux formant autanl de cheminées d'aspiration, ane ventilation très répartie et des plus efficace.

Appareils dans L'huile. - Pour les régulateurs devant ètre placés dans des milieux humides ou poussiéreux el sur des réseaux à tension relativement élevée, l'appareil, loujours à axe vertical, est placé dans l'huile contenue dans une cuve. Le refroidissement peul ètre naturel (cuve en tôle ondulée, fig. 19), ou forcé, soit par circulation d'eau à travers un serpentin noyè dans l'huile (fig. 20), soil par circulation de l'huile à travers un réfrigérant extérieur. L'enroulement induit peut ètre placé directement en série sur des réseaux dont la tension atleint 10.000 et même 15.000 volts, si la puissance est assez impor-

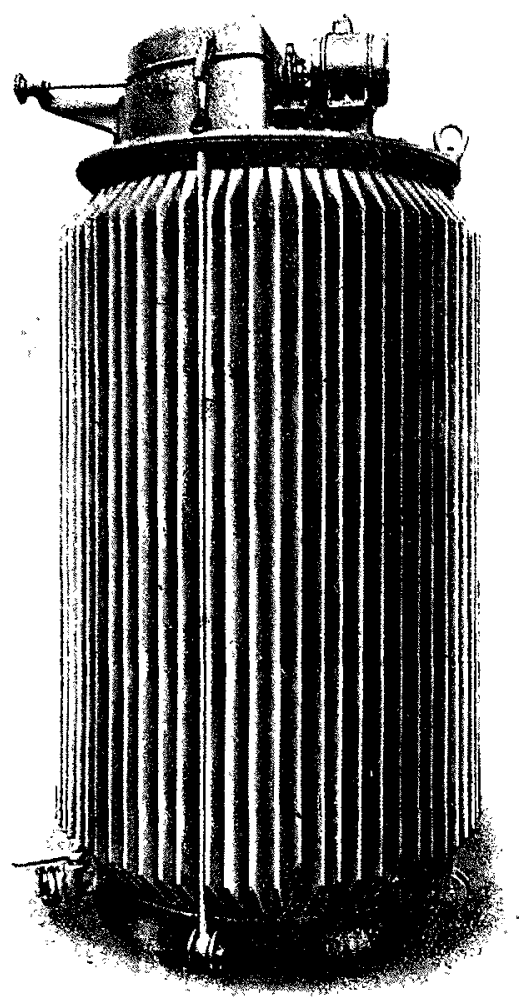

Fìg. 19. - Régulateur d'induction triphasé type ZMH à bain d'huile et refroidissement naturel.

$190 \mathrm{kVA} ; \pm 490$ volts par phase, 128 ampères, 50 périodes, à commande électrique à distance pour réglage de la tension primaire (5750 850 volts) du transformateur d'alimentation d'une commutatrice.

tante. Pour de telles tensions, en raison du grand nombre de conducteurs que nécessite l'enroulement et de la mauvaise utilisation en résultant, il est généralement préférable d'alimenter 'l'nducteur sous une tension plus faible, par exemple au moyen diun transformateur d'excitation spécial. Pour des réseaux à très haute tension, on peut intercaler également un transformateur série entre le réseau et l'enroulement induit. La disposition schématique est alors celle de la fig. 21 où, pour plus de clarté, on n'a représenté qu'une phase; $T_{1}$, est la transformateur série, $T_{2}$ le transformateur d'excitation et $R$ le régulateur d'induction, où, comme précédemment, $M$ caractérise l'enroulement inducteur alimenté par le secondaire du transformateur $\mathrm{T}_{2}$ et $\mathrm{F}$ l'enroulement induit alimentant l'un des enroulements de $\mathrm{T}_{\mathrm{I}}$ dont l'autre enroulement est intercalé directement en série sur le réseau. 


\section{IV) MISE EN SERVICE ET HORS SERVICE DES RÉGULATEURS D'INDUCTION BRANCHEMENT ET RÉGLAGE}

Lorsque le réseau n'est pas sous tension, il suffit, pour metle hors service un régulateur d'induction, de court-circuiter l'enroulemenl induit et de supprimer l'alimentation de l'inducteur. On pourrait aussi à la rigueur n'effectuer des mancuvres que sur l'enroulement inducteur, en court-circuitant cet enroulement après avoir coupé son alimentation, puisque dans ce cas, l'enroulement induit, parcouru par le courant du réseau ne représente qu'une self-induction faible, équivalente aux seuls flux de fuites du régulateur, mais on laisse ainsi subsister à peu près intégralement les pertes cuivre de l'appareil. Il est donc généralement prélérable d'adopter la première méthode, même si elle est plus coûteuse.

La Société Alsacienne a étudié un dispositif permettant de mettrẹ en sẹrvice oụ hors service les régulateurs d'induction sans male. En position 3, F est décourt-circuité et inséré dans le réseau; la résistance $R$ reste toujours intercalée dans le circuit d'excitation réduisant la tension induite dans le régulateur. Lorsqu'on atteint la position 4 , la résistance $\mathrm{R}$ est court-circuitée à son tour et l'appareil est en ordre de marche normale. Pour que le trouble apporté dans la tension d'utilisation aux positions 3 et 4 soit insensible à la mise en service, le courant ne peut être envoyé dans la bobine du contacteur d'enclanchement $\mathrm{C}_{1}$ que si le régulateur est préalablement amené dans sa position de" survoltage-dévoltage nul, c'est-à-dire dans la position où la tension d'utilisation reste égale à la tension d'alimentation. C'est le rôle de l'interrupteur $b$ et de l'inverseur automatique servant à l'alimentation du moteur de commande de la partie mobile du régulateur d'induction, celui-ci n'étant pas alors sous la dépendance du régulateur automatique de tension, supposé du système Thury dans la fig. 22, mais qu'on peut facilement imaginer d'un autre type connu. Dès que l'appareil est mis en service, le commutateur $b_{2}$, fermé en $b_{3}$, actionne la fermeture des contacts $a$, les contacts $b$ étant ouverts, et le moteur

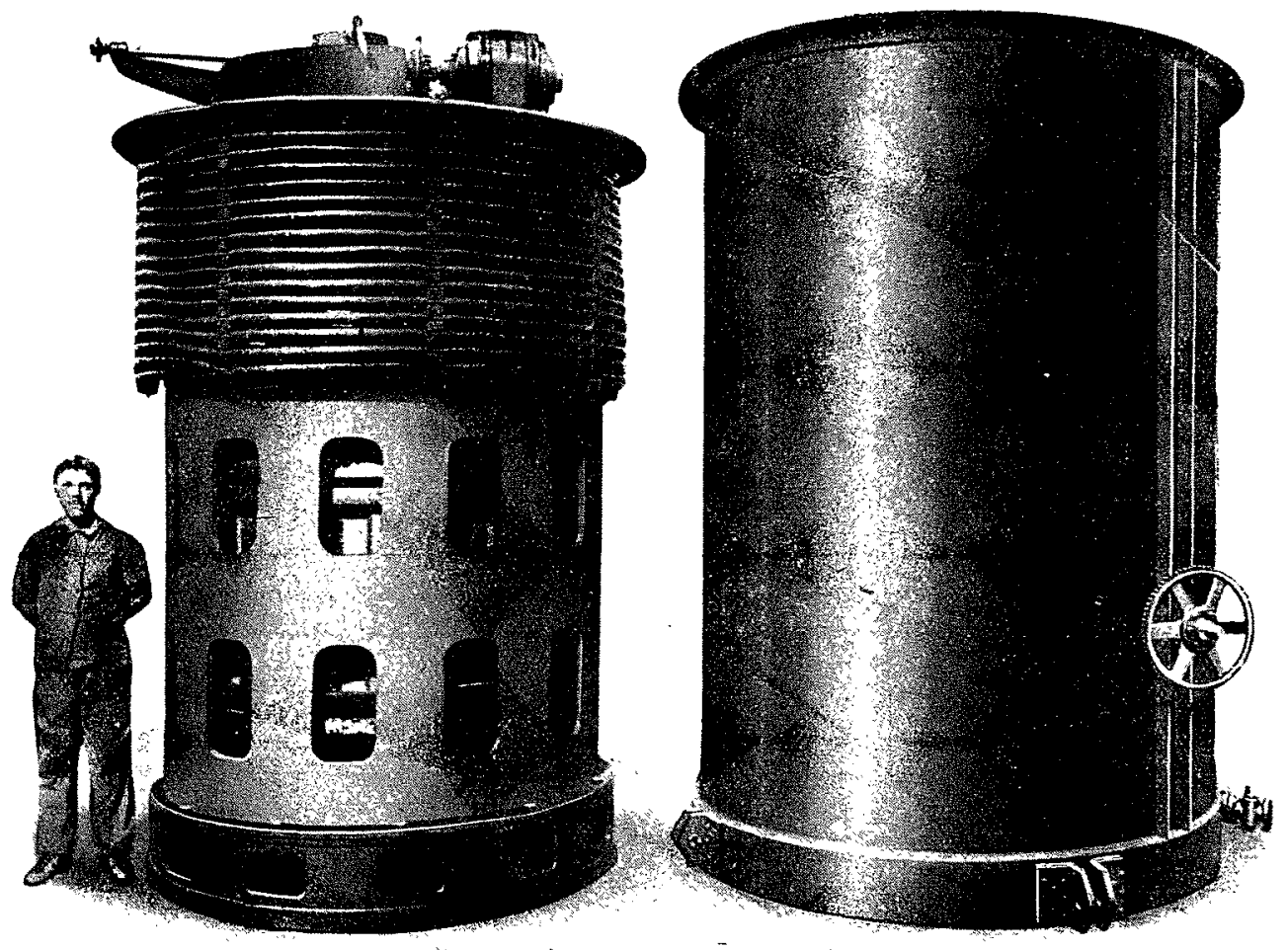

Fig. 20. - Régulateur d'induction triphasé type $\mathrm{Z} \mathrm{Y} \mathrm{H} \mathrm{O}$

à bain d'huile, à refroidissement artificiel par circulation d'eau, $1200 \mathrm{kVA} ; \pm 1000$ volts composés, 700 ampères, 50 périodes, à commande électrique à distance pour réglage de la tension d'un réseau, tension à régler variable entre 4500 et 5600 volts - tension réglée 5000 à 5500 volts.

interrompre la marche de l'exploitation et sans y provoquer des troubles sensibles. Le schéma de la fig. 22 représente l'installation qui comprend un système de deux interrupteurs tripolaires $A_{1}$ et $A_{2}$ supposés solidairement actionnés par un moteur-couple $B$, commandé à distance par l'interrupteur $J$. $A_{1}$ et $A_{2}$ occupent simultanément les positions successives $1,2,3$, et 4 . Dans la position 1 (régulateur hors service) $A_{1}$ courl-circuite l'enroulement induit $\mathrm{F}$ du régulateur d'induction. $\mathrm{A}_{2}$ coupe l'alimentaLion de l'enroulement inducteur M. Dans la position 2, F est toujours court-circuité, mais $M$ est déjà alimenté et pour empêcher les courants qui prement naissance dans $F$ et $M$ d'atteindre des valeurs correspondant au court-circuit, soit cinq à six fois la valeur normale, une résistance ohmique $R$, convenablement dimensionnée, limite ces courants au voisinage de la valeur nor- de commande du régulateur d'induction est alimenté par le régulateur Thury. Le réseau auxiliaire pour l'alimentation des

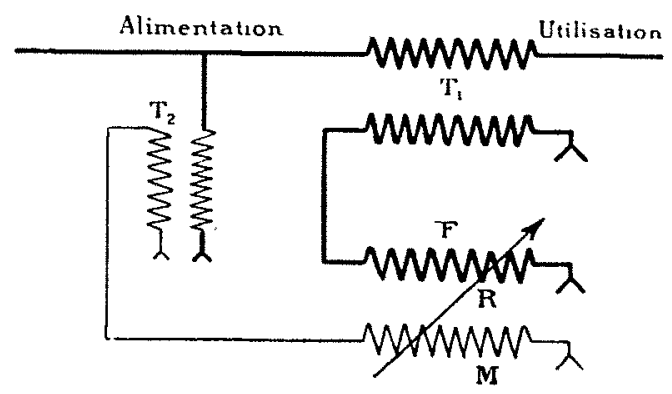

Fig. 21 
appareils de manceuvre et de réglage est supposé triphasé dans je cas de la fig. 22 ; il pourrait aussi bien être à courant continu.

La commande électrique du réglage de tension permet de corriger en une seconde des variations de tension pouvant alteindre un pour cent. Si une plus grande variation doit ètre corrigée dans le mème temps, la démultiplication entre les arbres du régulateur d'induction et du moteur de commande du réglage deviendrait si faible que ce moteur prendrait des dimensions exagérées. Le réglage s'obtient alors par servo-moteur à huile sous pression actionnant directement l'arbre du régulateur d'induction; on peut ainsi corriger des variations de $10 \%$ en une seconde.

Il est évident que le régulateur automatique employé doit comporter un asservissement empèchant le réglage de dépasser celui à réaliser et par suite les pompages. S'il s'agit d'obtenir une tension d'utilisation fonction de la charge du réseau, le régulateur tion. Un frein à commande électromagnétique esl égalenent placé sur l'arbre du moteur de commande, pour provoquer l'arrèt immédiat de celui-ci dès que son alimentation est coupee, car, bien que le mécanisme de commande du régulateur soil peu réversible, larrêt pourrait être plus ou moins rapide suivant lo sens dans lequel s'exerce le couple propre du régulateur d'induction.

\section{V) APPLICATIONS DES RÉGULATEURSD'INDUCTION}

Les différentes applications des régulateurs d'induction résultent des propriétés exposées précédemment; ces appareils permettent notamment de réaliser le réglage progressif et continu de la tension. On les emploie done:

$1^{\circ}$ Pour maintenir en un point d'utilisation une tension rigoureusement constante (par exemple sur un réseau d'éclairage)

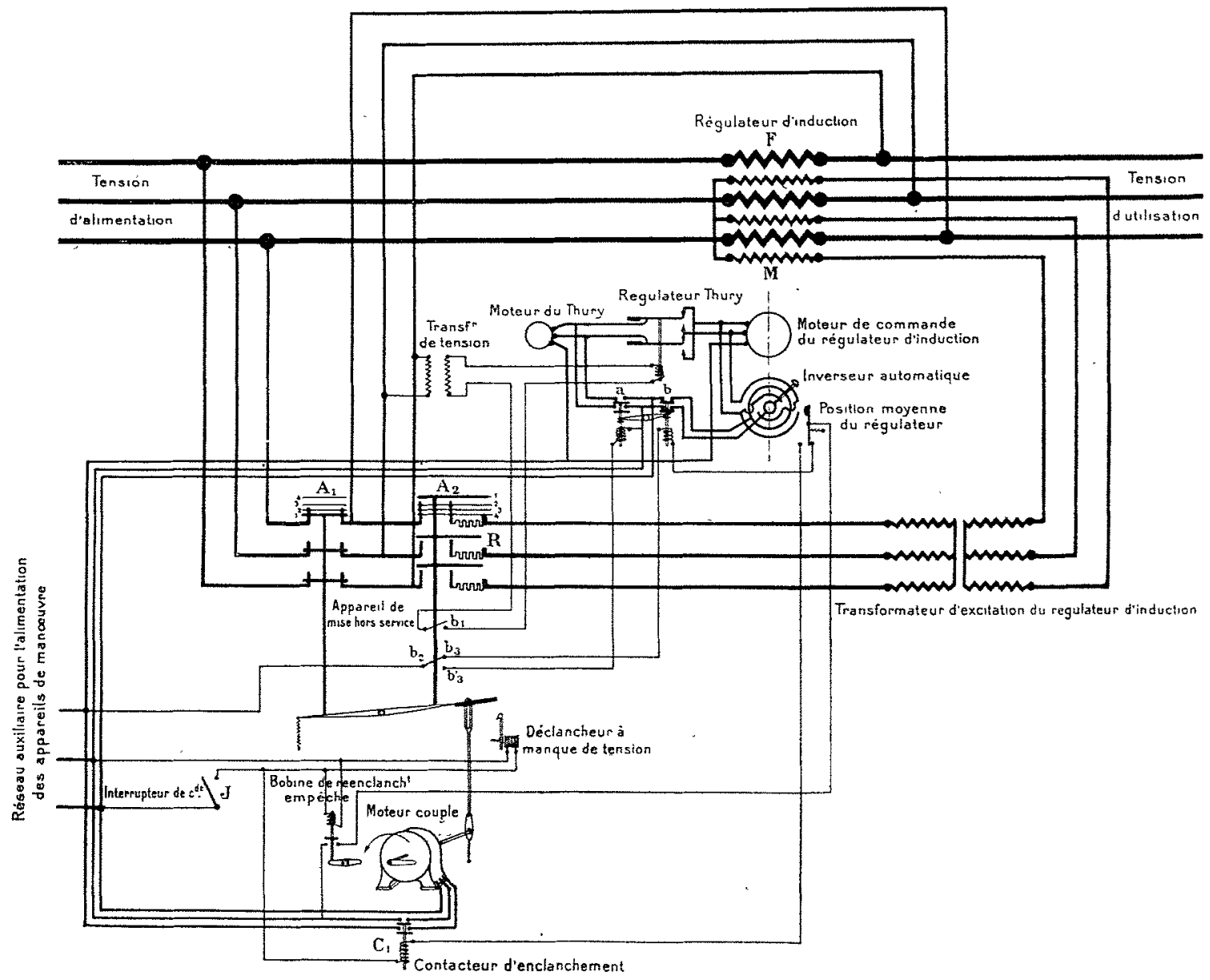

Fig. 22. - Schéma d'installation d'un régulateur d'induction avec régulateur Thury et dispositif de mise hors service commandé par moteur couple.

comporte une bobine de compoundage plaçant son point de réglage sous la dépendance du courant traversanl l'enroulement $F$ du régulateur d'induction.

Les régulateurs d'induction à réglage aulomatique de la Société Alsacienne sont également munis d'un dispositif de débrayage permettant d'effectuer au besoin le réglage à la main ; les poignées du dispositif de débrayage et de la manivelle de commande à main sont visibles sur la fig. 18 . On voit en outre sur la fig. 16 les contacts de fin de course coupant l'alimentation du moteur de commande pour un sens de rotation lorsque le régulateur d'induction est arrivé à la limite de sa course correspondant à ce sens. Ces contacts de fin de course sont actionnés par le secteur denté claveté sur l'arbre du régulateur d'induc- quelles que soient les variations de la charge ou de la tension au départ.

$2^{\circ}$ Inversement, pour régler la tension à l'origine d'une ligne ou d'un câble, la lension pouvant varier par exemple en raison de la charge pour compenser la chule en ligne. Nous avons dit que ce résultat peut être obtenu automatiquement par l'emploi d'un régulateur compoundé.

$3^{\circ}$ On emploie souvent les régulateurs d'induction aux points de couplage des réseaux. S'il s'agit seulement de réaliser en ces points un réglage de la tension pour effectuer le couplage de réseaux qui ne doivent pas être bouclés entre eux par ailleurs, on peut employer un régulateur d'induction simple, puisque les 
phases des tensions de ces réseaux peuvent s'ètablir indépendamment l'une de l'autre. Si les réscaux sont bouclés en plusieurs points, il laul employer un régulateur d'induction double. En choisissant alors convenablement la phase invariable de la tension réglable introduite par le régulateur d'induction double, on utilise cet appareil pour régler au point d'installation l'échange de puissance active ou de puissance réactive entre les deux réseaux. Si on place dans la ligne de liaison des réseaux deux survolteurs doubles en série, excités de façon à être le siège de tensions induites respectivement toujours en quadrature, on peut régler indépendamment l'échange de puissance active, par variation de la tension en quadrature avec la tension moyenne du réseau el l'échange de puissance réactive, par variation de la tension en phase avec la tension moyenne du réseau (1);

$4^{0}$ On intercale fréquemment des régulateurs d'induction du côté bagues des commutatrices dont on veut obtenir une tension du côté continu réglable dans de grandes limites. On sait que dans ce cas le réglage par bobine de self-induction el par variation du courant d'excitation ne peut guère dépasser $15 \%$, en raison soit du mauvais facteur de puissance sur le réseau altərnatif, soit du dimensionnement plus important imposé par l'échauffement pour certains organes de la commutatrice. Le régulateur d'induction s'emploie également avec des commutatrices à tension constante du côté contiuu, soit pour compenser les variations de tension du réseau alternatif, soit pour régler la charge de la commutatrice lorsque celle-ci fonctionne en parallèle avec d'autres machines. Dans le cas de distributions à trois fils dans lesquelles le fil neutre de côté continu est relié au point neutre du réseau alternalif (transformateur en général), le régulateur d'induction doit comporter autant d'enroulements qu'il y a de bagues du côté alternatif de la commutatrice, de façon à produîr e

(1) Voir à se sujet le Bulletin de la Société Alsacienne de Constructions Mécaniques d'octobre 1925 et le compte rendu de la communication faite par $M$. Roth à la $1^{\text {re }}$ section de la Conférence Internationale des grands Réseaux de juin 1925. des variations symétriques et simultanées du potentiel de chaque bague par rapport à celui du point neutre.

$5^{\circ}$ Les grandes stations centrales possèdent aujourd'hui des alternateurs spéciaux pour l'essai et la mise sous tension progressive des câbles avant la connexion de ceux-ci aux rails d'alimentation. Il faut dans ce cas prévoir éventuellement les moyens de mise en phase nécessaires. Le régulateur d'induction peut également être utilisé dans ce cas; on le fait simple ou double selon qu'il doit servir uniquement à l'essai, ou également au couplage ; il n'y a pas alors de mise en phase à effectuer;

$6^{\circ}$ Dans les laboratoires, le régulateur d'induction rend également de grands services. Pour l'étalonnage de wattmètres ou de compteurs, on utilise le déphasage produit par le régulateur d'induction simple pour vérifier les indications de ces appareils sous différents facteurs de puissance, sans qu'il soit nécessaire de produire les charges correspondantes. Le régulateur d'induction simple permet en effet d'obtenir une résistance ohmique variable, par exemple en partant de la tension d'utilisation, un courant constant de phase variable par rapport à la tension d'alimentation (on alimente donc pour l'étalonnage le fil fin du wattmètre par la tension d'alimentation et on envoie le courant déphasé par rapport à cette tension dans le gros fil).

$7^{\circ}$ Dans les fours électriques, le régulateur d'induction monophasé ou polyphasé permet d'obtenir une tension variable, à partir d'une valeur sensiblement nulle si cela est nécessaire

Dans les usines modernes d'ammoniaque, on emploie également les régulateurs d'induction pour le réglage du chauffage des tubes de catalyse, etc...

Comme on le voit, les applications des régulateurs d'induction sont nombreuses et variées et ces appareils jouent un rôle important dans le transport et la distribution de l'énergie électrique.

\section{J. BERGER.}

\section{Conditions de sécurité de la Traction électrique}

On peut dire que la traction électrique des trains de grandes lignes est encore à son aurore, et qu'elle sera, dans l'avenir, l'objet de maint perfectionnement.

Une question, déjà agitée à plusieurs reprises, est celle de savoir s'il convient de confier à un seul homme la conduite d'une locomotive électrique, ou s'il est nécessaire de prévoir un mécanicien de rechange on tout an moins un aide, capable d'arrêter le convoi en cas de défaillance du titulaire. Avec beaucoup de raison on observe que le deuxième mécanicien n'aurait rien à faire, mais avec non moins de raison on observe que le titulaire peut être frappé de syncope et laisser entrevoir les terribles catastrophes qui pourraient survenir en pareil cas.

En tous pays, les mécaniciens de locomotive sont soumis à une sélection rigoureuse, résultant d'examens minutieux portant sur les connaissances techniques indispensables, puis sur la résistance physique, l'émotivité, la perfection de la vision, en particulier au point de vue du daltonisme, celte étrange infirmitè qui fait confondre le rouge et le vert, précisément les couleurs des signaux. on peut dire que partout les mécaniciens sont des hommes d'élite ; toutefois, comme chacun de nous, ils peuvent ètre frappès de ces affections subites, parfois mortelles, la rupture d'anévrisme, la syncope, la congestion. Au point de vue accidents, ceux qui conduisent les locomotives électriques sont moins exposés que le conducteur d'une machine à vapeur et ils se trouvent dans des conditions de confortable bien supérieures. Placés dans un local fermé, ils sont complètement à l'abri des intempéries, et leur vue n'est pas gênée par la fumée et la vapeur, notamment dans les tunnels.

Quoique d'une autre nature, les accidents pouvant survenir à la machine même, et pouvant par contre-coup atteindre le mécanicien sont de mème importance sur la locomotive électrique et sur celle à vapeur. L'explosion de la chaudière à vapeur est très rare, et pour ma part je ne l'ai entendu qu'une fois, entre SaintLazare et le tunnel des Batignolles, à Paris, il y a une trentaine d'années. Il arrive plus fréquemment des ruptures de tubes, produisant une fuite de vapeur qui peut brûler le chauffeur au moment de l'ouverture de la porte du foyer. Sur la locomotive électrique peuvent se produire des courts-circuits, particulièrement redoutables par suite de la hạute tension employée. L'expé 